\title{
Enhancing Adolescents' Environmental Responsibility Through Outdoor Recreation Activities
}

\author{
Aglaia Zafeiroudi \\ Ph.D., Educational Staff, \\ University of Thessaly, Greece
}

DOI: https://doi.org/10.36941/ajis-2020-0110

\section{Abstract}

Spending time outdoors fosters a sense of connectedness and empathy with nature and has a clear effect on attitudes and behaviour towards the environment. The purpose of this study was to examine the impact of an outdoor activities intervention program on adolescents' sense of responsibility towards the environment. Participants included 262 adolescents (135 girls and 127 boys) aged 13-17 years who were divided into experimental $(n=141)$ and control groups $(n=121)$. The 15-day outdoor intervention program included trekking, mountain biking, orienteering, archery, ropes courses, canoeing on a lake, via ferrata rock climbing, night hiking, and overnight camping. Participants completed an Environmental Responsibility questionnaire (Zafeiroudi E Hatzigeorgiadis, 2013) before and after participation in the activities program. The questionnaire included five scales that focused on perceptions of information on environmental issues, environmental cognitive and affective beliefs, predisposition for environmental action, and environmentally responsible behaviour. Analysis of variance with repeated measures revealed improved scores amongst adolescents who participated in the intervention program, notably within the specific scales used to evaluate perceived information, affective beliefs, predisposition for individual action, and predisposition for encouraging others. A follow-up analysis of covariance revealed that participants in the experimental group scored higher overall than did those in the control group. Taken together, these findings add to the current literature focused on psychosocial and behavioural mechanisms associated with environmental awareness. Our findings also suggest that outdoor recreational activities may be an effective supplement and a more challenging alternative to programs currently provided by environmental and active leisure associations.

Keywords: Psychosocial Behaviour, Leisure, Intervention Program, Environmental Education, Physical Education

\section{Introduction}

Many recent findings indicate that our natural environment is in significant danger, as pollution currently consumes many of the world's most beneficial resources. In the face of environmental deterioration, concerned individuals have focused on efforts to promote resource conservation. These efforts include blue economy, environmental awareness programs, and other initiatives designed to address significant environmental problems currently threatening the population worldwide.

\section{$1.1 \quad$ Background}

Over the past decade, humans have been involved in careless and detrimental activities that have resulted in the destruction of many natural habitats (Erdogan, 2015). These activities have generated 
numerous environmental problems that are harmful to society as a whole. Most researchers believe that we need to develop an environmentally conscious and responsible society whose members are focused on alleviation of these imminent problems (Harbrow, 2019). Towards this end, there are early signs suggesting that environmental education programs have a significant impact with respect to improving society. Some of these early initiatives include programs that provide students with outdoor experiences that are preferable to classroom-based conceptual instruction. Specifically, outdoor experiences may enhance student interest in developing more positive behaviours with respect to the environment and may promote environmental responsibility (Collado, Corraliza, Staats \& Ruiz, 2015; Zafeiroudi \& Hatzigeorgiadis, 2014a; Palmberg \& Kuru, 2000; Sivek, 2002).

Studies published by Mackenzie et al., (2018) and Erdogan (2015) focused on the Summer Environmental Education Program (SEEP) and its impact on environmental literacy, including student behaviour, affect, environmental knowledge, and skills, amongst other factors. These parameters were evaluated in students who participated in the SEEP study who represented a significant segment of the adolescent population. The results of the SEEP study revealed that students' environmental knowledge, attitudes, sensitivity, responsibility, and intention all increased after a significant intervention. Subsequent research from Mackenzie et al. (2018) revealed that outdoor education programs provide personal as well as social benefits that had not been realized previously. Specifically, Mackenzie et al. (2018) argued that the rationale for the outdoor education in the United Kingdom grew from accepted practices that lacked a philosophical foundation. As such, the rationales for these programs focused only on traditional assumptions regarding adventurous outdoor activities and did not provide a clear or coherent representation of the extent or utility of outdoor education.

Although insufficient attention has been paid to environmental responsibility, several studies, such as that of Blythe and Harre (2020), provide a strong rationale for the development of a stronger student knowledge base, specifically one that includes ecological concepts, wilderness philosophies, and environmental issues. These concepts can be taught through effective training and by the use of other significant teaching methods. For example, findings from a study conducted in Finland indicated that outdoor activities were associated with a high likelihood of developing empathic relationships with nature and connectedness to the natural world (Rantala \& Puhakka, 2020). Also, there is an interaction between families and young people. Similarly, Zafeiroudi and Hatzigeorgiadis (2014b) evaluated the relationship between the frequency of participation in outdoor recreation and scores on a Responsible Environmental Behaviour Scale amongst residents of the Attica municipality in Greece. These researchers found that respondents who participated in outdoor recreation on a more frequent basis scored higher with respect to both group and individual actions compared with those who did not participate in these activities. Furthermore, Harbrow (2019) reported that increased levels of environmental awareness, action, and concern can be realized through significant participation in most recreational adventure activities. However, despite the clear findings from these important studies, the impact of outdoor activities on environmental responsibility observed amongst adolescents remains unclear. Specifically, there is little to no information available that focuses on use of specific scales to measure environmental awareness, interrelationships, and changes of behaviour and attitudes towards environmental responsibility amongst younger participants. This is particularly notable amongst those who participated in outdoor recreational activities but who have not yet experienced a program specifically designed to promote outdoor education.

\subsection{Environmental behaviours amongst adolescents and attitudes towards environmental responsibility}

Concern regarding environmental responsibility is increasing on a daily basis; specific attention is currently focused on environmental behaviours. According to Kukkonen, Karkkainen, and Keinonen (2018), environmental responsibility is a key theme associated with education designed to promote sustainability. As a group, these themes feature dimensions that include attitudes and sensitivity towards the environment and environmentally friendly actions, as well as knowledge, environmental 
awareness, and participation. Based on these findings, Kukkonen et al. (2018) focused on relationships amongst factors used to predict sustainable behaviours and environmental responsibility. This study includes a more substantial focus on psychosocial behaviours, including adherence to social norms, feelings of guilt, awareness of specific problems, and internal attribution. Notably, while the intention to act can clearly modify a given behaviour, the influence of knowledge on the same behaviour was found to be both complex and indirect.

Kukkonen et al. (2018) argued that one cannot consider behaviour without specific reference to attitude. This argument implies that any observations related to pro-environmental behaviours go hand-in-hand with their relationships to situational parameters, values, beliefs, and environmental attitudes. Results from earlier studies suggested that it is indeed possible for adolescents to develop environmentally focused behaviours (Singh, Goyal \& Jain, 2017). These researchers published the results of an experimental study that aimed to establish the effectiveness of an active learning program and of traditional teaching methods used to promote environmental responsibility amongst adolescents in India. Specifically, these researchers found that active learning played a significant role in environmental education and served to motivate behaviours that promote conservation, preservation, and protection of the environment. Some of the activities incorporated in environmental education programs that are overall closely associated with environmental behaviours include games, class discussions, small group projects, demonstrations, debates, information gathering, site visits, practical work, dramatizations, guest lectures, and outdoor studies.

Singh et al. (2017) stated emphatically that the locus of control for environmental behaviours is broad and can present with multiple dimensions. Considering the fact that adolescents are largely still in school, observable variables would be unlikely to depart significantly from the locus of control, environmental sensitivity, knowledge with respect to environmental action, perceived skill, beliefs, attitudes, and psychological sex roles. Based on these observations, perceived skill might be a focal point with respect to responsible behaviours (Heers, Van Klaveren, Groot, \& Maassen van den Brink, 2016). However, both the individual and group loci of control may lag behind perceived skills with respect to environmental action.

1.3 Human factors that can be influenced by outdoor activities to support the development of environmental responsibility amongst adolescents.

Amongst other factors that contribute to the development of environmental responsibility through outdoor activities, Thatcher, Waterson, Todd, and Yeow (2018) noted that human factors need to be regarded as a high priority. Amongst adolescents, human factors examined include shared values that can promote new designs and models. The concept of shared values describes social and ecospheric responsibility with a focus on environmental awareness. This can best be attained via education on sustainable systems and sociotechnical principles associated with environmental responsibility and sustainability. Furthermore, there is an additional focus on ethics as a primary human value. Based on this concept, Thatcher et al. (2018) drew attention to unjust social relationships and the inequitable distribution of available resources amongst members of the global society. Of note, only very rarely do individuals willingly assume the duty of selecting choices that enhance environmental responsibility without significant guidance provided by relevant ethics and moral practices.

While classroom theories include some reference to ethics, outdoor activities are required to convert these theoretical constructs into functional practices (Reis, Salvo, Ogilvie, Lambert, Goenka, Brownson, \& Lancet Physical Activity Series 2 Executive Committee, 2016). Real-world experience is therefore of major importance and needs to be maintained as a focus while at the same time addressing ethical practices associated with human health and well-being (Lubans, Richards, Hillman, Faulkner, Beauchamp, Nilsson, \& Biddle 2016). In addition to ethics, it is also critical to embrace individual as well as environmental diversity on a global scale. Sathe (2018) reported that adolescents need to have a working comprehension of parameters that define individual diversity from an early age in order to have the means to appreciate and to respect the needs of people outside their personal social spheres. 

amongst adolescents

Studies that feature recreational activities and their specific relationships with environmental responsibility have promoted a broad evaluation of established psychosocial systems. Lubans et al. (2016) asserted that it is necessary to consider both eudemonic and hedonic perspectives while considering the impact of physical activities and their capacity to enhance adolescents' well-being. Several theoretical frameworks have been presented to explain the mechanisms used to satisfy the psychosocial need for self-acceptance, autonomy, connectedness, purpose, and environmental mastery. Lubans et al. (2016) noted that recreational activities provide an opportunity for social interaction; these activities have been associated with relatedness and a mastery of one's physical domain, as well as with independence and self-perception. These parameters necessarily increase adolescents' focus on their natural environment. Consistent participation in recreational activities promotes improved self-efficacy, a principle that can generalized to include the physical self-concept and self-esteem. Collado et al., (2015) also indicated that participation in outdoor activities affect children's engagement in pro-environmental behaviour.

The concepts established from these findings are largely grounded in a theory that postulates that human beings are naturally inclined to be influenced, reassured, or fascinated by their surroundings. Some studies have identified links between the degree of children's and adolescents' outdoor experiences and their empathy with nature (Cheng \& Monroe, 2012; Stevenson, Peterson, Carrier, Strnad, Bondell, Kirby-Hathaway, \& Moore, 2014). These findings imply that connectedness with nature is associated with positive mental health outcomes and as such may also have a positive impact on environmental responsibility. Taylor, Clark, and Gilliland (2018) explored health-related behaviours associated with planned physical activities amongst adolescents from the Ontario of Canada. Several of the selected communities included in this study were focused on themes that included parental support, competing responsibilities, and participation in physical activity; the findings obtained provided a means to link health-related behaviours associated with physical activities to environmental conditions. Taylor et al. (2018) noted that behaviour was shaped primarily by the social constructs associated with the local environment; this finding may be related to the fact that activities commonly take place within the significant confines of communities, neighborhoods, and families. These findings ultimately promoted recommendations for further exploration of social and environmental factors as well as physical activities that might be more appealing to school-aged adolescents. Of note, several researchers have identified a strong relationship between participation in outdoor recreation and proenvironmental behaviours (Zafeiroudi \& Hatzigeorgiadis, 2014b; Larson, Whiting, \& Green, 2011).

Although several published studies have explored the relationships between environmental variables and children's involvement in outdoor recreational activities via both outdoor education and outdoor adventure education, there are very few studies that examine the effects of outdoor recreation directly on environmentalism in the absence of a concomitant environmental education program.

This research study aimed to examine the impact of outdoor activities on adolescents' sense of environmental responsibility based on their participation in an outdoor recreational activities program. The research focused on several objectives, including an evaluation of environmental behaviours amongst adolescents and their relationship to environmental responsibility, a statistical evaluation of five key parameters that might be influenced by adolescents' participation in outdoor recreational activities designed to support environmental responsibility, and an examination of the factors that influence adolescents' sense of environmental responsibility that results from structured outdoor recreational programs. 


\section{Methods}

\subsection{Participants}

The study enrolled 262 participants (135 girls and 127 boys) who were between 13 and 17 years of age. One hundred seventy-five participants were students enrolled in secondary school and 87 were in high school. Of this group, 89 children (34\%) resided with their families in a village, 55 children (21\%) resided in a suburb of a large city, and 118 children $(45 \%)$ resided in a large city. One hundred thirtythree of the study participants (50.8\%) resided in detached houses or maisonettes, while $129(49.2 \%)$ lived in apartments. All participants' homes scored equally when evaluated for access to green landscapes that could be viewed from the kitchen, bedroom, and living room.

\subsection{Instrumentation}

Participants completed an Environmental Responsibility instrument (Zafeiroudi \& Hatzigeorgiadis, 2013) which included the following five scales: perceived information for environmental issues, environmental cognitive beliefs, environmental affective beliefs, predisposition for environmental actions, and environmentally responsible behaviour. The perceived information scale was used to assess the extent to which participants believed that they were informed about general environmental issues. The scale included consisted of 11 items (e.g., How informed do you believe that you are about climate change?). Responses were provided using a 5 -point Likert scale ( $1=$ not at all to $5=$ very much). The environmental beliefs scales included dimensions of both cognitive and affective beliefs. The cognitive scale included 7 items that were used to evaluate the extent to which an individual focused on ways to protect the environment by engaging in helpful actions (e.g., We should learn to identify products that do not pollute the environment). The affective scale included 7 items that were used to assess the impact of events and actions associated with environmental protection on emotional wellbeing (e.g., I feel happy when people try not to waste energy). Responses were provided using a 5-point Likert scale $(1=$ strongly disagree to $5=$ strongly agree). The predisposition for environmental action scale included 8 items used to assess this attribute as a prelude to pro-environmental behaviour. This scale included two factors: a predisposition for individual environmental action that included 5 items (e.g., I am willing to travel by bus or bicycle in order to reduce air pollution) and a predisposition for encouraging others to participate in environmental action (e.g., I am willing to go door-to-door to ask others to recycle). Responses were provided using a 5 -point Likert scale $(1=$ strongly disagree to $5=$ strongly agree). The environmentally responsible behaviour scale was used to assess individual behaviour towards the environment. This scale also included two factors: individual environmental action that was assessed with 7 items (e.g., How frequently do you use products that are provided in recyclable packages or containers?) and group environmental action with 3 items (e.g., How frequently do you participate in events related to environment?). Responses were provided using a 5-point Likert scale $(1=$ never to $5=$ every day $)$.

\subsection{Procedure}

The study was conducted simultaneously at four summer camps located in different districts within Greece and included participants from the central (Karpenisi and Pertouli), southern (Parnitha) and northern (Chalkidiki) regions. An approximately equal number of participants (25 - 35 adolescents) were recruited from each summer camp. Participants were divided randomly into experimental and control groups. All adolescents participated voluntarily in the program upon written parental consent.

\subsubsection{Control group.}

The control group included 121 adolescents (aged 13-17 years) who completed the same questionnaire 
as did participants in the experimental group. Participants in the control group completed the questionnaire twice, once at the beginning and again at the end of the camp period. The control group followed a conventional summer camp program that included sports, pool-based aquatic activities, board games, music/dance/theatre/painting activities, watching films, and participation in crafts, cooking, and social events.

\subsubsection{Experimental group.}

This experimental group included 141 adolescents (aged 13-17 years) who completed the same questionnaire as did participants in the control group. Participants in the experimental group completed the questionnaire twice, once before and once again after participation in the specific intervention program. Adolescents enrolled in this program remained at the camp for the entire 15-day period and participated in all of the interventional program activities. Adolescents who did not complete the 15-day camp period were excluded from the study.

\subsection{Intervention program}

The intervention program included primarily outdoor recreation activities. The activities provided as part of the intervention program included trekking, mountain biking, orienteering, archery, ropes courses, lake canoeing, via ferrata rock climbing, night hiking, and overnight camping. The duration of the camp period was 15 days with approximately 6-10 hours of active participation each day. The activities were designed in accordance with participant age, characteristics, and experiences. The program included outdoor recreation activities only, with no direct environmental education program. The staff included facilitators who specialized in outdoor recreational activities.

\section{Results}

\subsection{Descriptive statistics and baseline differences}

Descriptive statistics that detail the participants' responses before and after the intervention are presented in Table 1. All participants achieved high scores on cognitive and affective environmental beliefs scales both before and after the intervention. By contrast, most participants achieved low to moderate scores on scales focused on predisposition individual action and predisposition for encouraging others on initial assessment and achieved higher scores in the final evaluation. The environmentally responsible behaviour scale was evaluated before the intervention only. Moderately high scores were achieved for scales focused on individual environmental action, while lower scores were achieved for scales focused on group environmental action.

Table 1. Descriptive statistics and reliability scores $(\mathrm{N}=262)$

\begin{tabular}{lcccccc}
\hline & \multicolumn{3}{c}{ Pre } & \multicolumn{3}{c}{ Post } \\
\cline { 2 - 7 } Perceived information & Mean & S.D. & Alpha & Mean & S.D. & Alpha \\
\cline { 2 - 7 } Cognitive & 3.58 & 0.67 & .83 & 3.62 & 0.70 & .87 \\
Affective & 4.23 & 0.55 & .74 & 4.20 & 0.57 & .75 \\
Predisposition for individual action & 4.03 & 0.66 & .83 & 4.09 & 0.67 & .85 \\
Predisposition for encouraging others & 3.92 & 0.79 & .66 & 4.02 & 0.96 & .84 \\
Individual environmental action & 2.95 & 1.00 & .73 & 3.03 & 1.00 & .86 \\
Group environmental action & 3.44 & 0.74 & .68 & - & - & - \\
\hline
\end{tabular}




\subsection{Correlations}

Correlations between all variables evaluated in this study are presented in Table 2. Correlations between the initial measurements are those shown under the diagonal; values presented over the diagonal are correlations between final measurements. Responses to questions that focused on affective environmental beliefs were most highly correlated with those associated with cognitive beliefs in both the initial and final measurements. The next highest correlations were found between those focused on predisposition for individual action and affective cognitive beliefs from responses to the initial questionnaire and between perceived information and cognitive beliefs from responses on the final evaluation. Comparatively lower correlations were identified for comparisons between group environmental actions and cognitive beliefs based on responses to the initial evaluation and predisposition for individual and group environmental action in the final measurement. Overall, our findings included significant positive correlations between all of the aforementioned attributes.

Table 2. Correlations between variables for pre intervention (under diagonal) and post intervention (over diagonal)

\begin{tabular}{lccccccc}
\hline & 1 & 2 & 3 & 4 & 5 & 6 & 7 \\
\hline Perceived information & - & $.52^{* *}$ & $.51^{* *}$ & $.28^{* *}$ & $.27^{* *}$ & $.46^{* *}$ & $.40^{* *}$ \\
Cognitive & $.44^{* *}$ & - & $.66^{* *}$ & $.37^{* *}$ & $.38^{* *}$ & $.43^{* *}$ & $.24^{* *}$ \\
Affective & $.37^{* *}$ & $.61^{* *}$ & - & $.44^{* *}$ & $.41^{* *}$ & $.41^{* *}$ & $.25^{* *}$ \\
Predisposition individual action & $.34^{* *}$ & $.55^{* *}$ & $.59^{* *}$ & - & $.37^{* *}$ & $.26^{* *}$ & $.15^{*}$ \\
Predisposition encouraging others & $.25^{* *}$ & $.34^{* *}$ & $.37^{* *}$ & $.43^{* *}$ & - & $.36^{* *}$ & $.36^{* *}$ \\
Individual environmental action & $.50^{* *}$ & $.46^{* *}$ & $.41^{* *}$ & $.46^{* *}$ & $.42^{* *}$ & - & $.40^{* *}$ \\
Group environmental action & $.37^{* *}$ & $.20^{* *}$ & $.21^{* *}$ & $.27^{* *}$ & $.44^{* *}$ & $.40^{* *}$ & - \\
\hline
\end{tabular}

${ }^{*} \mathrm{P}<.05,{ }^{* *} \mathrm{p}<.01$

\subsection{Baseline differences in pre-intervention scores}

One-way multivariate analysis of variance (MANOVA) was calculated to examine the differences between the experimental and control groups at first evaluation with respect to factors associated with perceived information, cognitive beliefs, affective beliefs, predisposition for individual action, and predisposition for encouraging others. The analysis revealed a significant group-by-time interaction effect $\left[F(5,256)=1.95, p=.08\right.$, and partial $\left.\eta^{2}=.04\right]$. Examination of the univariate effects revealed that the differences were significant with respect to perceived information $[F(1,260)=4.60, p<.05$, partial $\left.\eta^{2}=.02\right)$, cognitive beliefs $\left[F(1,260)=2.86, p=.09\right.$, and partial $\left.\eta^{2}=0.01\right]$, and predisposition for individual action $\left[F(1,260)=6.31, p<.05\right.$, and partial $\left.\eta^{2}=.02\right]$. Overall, the experimental group scores were higher than those of the control group. There were no significant effects associated with affective beliefs $[F(1,260)=2.21, p=.14]$ or with a predisposition for encouraging others $[F(1,260)=0.02, p=$ $.89]$. Overall, the results suggested a tendency towards higher baseline scores amongst those in the experimental group compared to those measured amongst participants in the control group.

To examine the impact of the intervention program, two types of analyses were performed. Repeated measures of analysis were performed to examine the differences between pre- and postintervention scores reported for both groups. Likewise, analysis of covariance (ANCOVA) was performed to examine differences in post-intervention with respect to differences observed in preintervention scores.

\subsection{Repeated measures}

A two-way ANOVA with one repeated factor (time: pre- and post-intervention) and one independent variable (group: experimental and control) was conducted to test for differences in perceived 
information, cognitive beliefs, affective beliefs, predisposition for individual action, and predisposition for encouraging others between the two groups and also before and after the intervention. This analysis revealed a significant multivariate interaction between group and time $[F(5,256)=8.76, p<.01$, and partial $\left.\eta^{2}=.14\right]$. Examination of the univariate effects revealed that the interaction was significant for perceived information $\left[F(1,260)=31.41, p<.01\right.$, and partial $\left.\eta^{2}=.01\right]$, for affective beliefs $[F(1,260)=$ $8.87, p<.01$, partial $\left.\eta^{2}=.03\right]$, for the predisposition for individual action $[F(1,260)=6.94 p<.01$, partial $\left.\eta^{2}=.03\right]$ and for the predisposition for encouraging others $\left[F(1,260)=7.10, p<.01\right.$, partial $\left.\eta^{2}=.03\right]$, but not for cognitive beliefs $[F(1,260)=0.67, p=.41]$. Pairwise comparisons for perceived information revealed that scores for the experimental group increased after the intervention $(p<.01)$, whereas scores for the control group decreased significantly $(p<$. ol). Pairwise comparisons for affective beliefs revealed that scores for the experimental group increased after the intervention $(p<.01)$, whereas scores for the control group underwent no significant change. Pairwise comparisons for the predisposition for individual action revealed that scores for the experimental group increased after the intervention $(p<.01)$, whereas scores for the control group underwent no significant change. Finally, pairwise comparisons for the predisposition for encouraging others revealed that scores for the experimental group increased after the intervention $(p<.01)$, whereas scores for the control group underwent no significant change. Mean scores for both groups are presented in Table 3 .

Table 3. Descriptive statistics for the experimental and control groups on the pre- and postintervention assessments.

\begin{tabular}{lcccc}
\hline & \multicolumn{2}{c}{ Experimental group } & \multicolumn{2}{c}{ Control group } \\
\cline { 2 - 5 } & Pre & Post & Pre & Post \\
\cline { 2 - 5 } Perceived information & $3.66 \pm 0.59$ & $3.82 \pm 0.58$ & $3.48 \pm 0.75$ & $3.39 \pm 0.75$ \\
Cognitive & $4.28 \pm 0.44$ & $4.27 \pm 0.52$ & $4.17 \pm 0.65$ & $4.12 \pm 0.61$ \\
Affective & $4.09 \pm 0.58$ & $4.21 \pm 0.61$ & $3.96 \pm 0.73$ & $3.94 \pm 0.71$ \\
Predisposition for individual action & $4.04 \pm 0.72$ & $4.25 \pm 0.98$ & $3.79 \pm 0.85$ & $3.74 \pm 0.87$ \\
Predisposition for encouraging others & $\mathbf{2 . 9 5 \pm 1 . 0 2}$ & $3.13 \pm 1.02$ & $\mathbf{2 . 9 4} \pm 0.97$ & $2.90 \pm 0.96$ \\
\hline
\end{tabular}

\subsection{ANCOVA}

ANCOVA was performed to evaluate differences between the pre-intervention assessment scores of the control and experimental groups for perceived information, cognitive beliefs, and the predisposition for individual action. One-way ANCOVA was performed with the pre-intervention evaluation as the covariate and group as the dependent variable. For perceived information, this analysis revealed that the effect of group was significant $\left[\mathrm{F}(1,261)=41.21, p<.01\right.$, and partial $\left.\eta^{2}=.14\right]$. Examination of the estimated means for all pre-intervention scores suggested that experimental group scored higher than control group. For cognitive beliefs, this analysis revealed that the effect of group was not significant $[\mathrm{F}(1,261)=1.85$, and $p=.17]$. Examination of estimated means also revealed no significant effect. For affective beliefs, this analysis revealed that the effect of group was significant [ $\mathrm{F}$ $(1,261)=12.69, p<.01$, and partial $\eta^{2}=.05$ ]. Examination of estimated means for pre-intervention scores suggested that the experimental group scored higher than the control group. For the predisposition for individual action, this analysis revealed that the effect of group was significant $[\mathrm{F}(1,261)=12.69, p<$ .01 , and partial $\eta^{2}=.05$ ]. Examination of estimated means for pre-intervention scores suggested that the experimental group scored higher than the control group. Finally, for the predisposition for encouraging others, this analysis revealed that the effect of group was significant $[\mathrm{F}(1,261)=8.18, p<$ .01, partial $\eta^{2}=.03$ ]. Examination of estimated means of pre-intervention scores suggested that the experimental group scored higher than the control group. 
Table 4. Ancova analysis

\begin{tabular}{lcc}
\hline & Experimental group & Control group \\
\cline { 2 - 3 } & Mean & Mean \\
\cline { 2 - 3 } Perceived information & 3.75 & 3.47 \\
Cognitive beliefs & 4.23 & 4.17 \\
Affective beliefs & 4.17 & 3.99 \\
Predisposition for individual action & 4.18 & 3.82 \\
Predisposition for encouraging others & 3.12 & 2.92 \\
\hline
\end{tabular}

\section{Discussion}

This research aimed to establish the impact of outdoor activities on factors associated with environmental responsibility in a cohort of school-age adolescents. The research was based on a number of assumptions, including the premise that the adolescents who participated in the study were a representative segment of the active population. Observable parameters were captured with objectives that focused on environmental behaviours, structured recreational activities, and the scope of environmental responsibility. Amongst other observable characteristics, the study identified five key parameters that may be used to evaluate the impact of outdoor activities on the development of environmental responsibility. The five parameters evaluated in this study include perceived information, cognitive beliefs, affective beliefs, the predisposition for encouraging others, and the predisposition for individual action. All parameters surpassed an affirmative mean of 2.5 in both the control and the experimental groups. However, significantly higher scores were achieved overall by participants in the experimental group who were provided with the opportunity to interact with the environment via the specific intervention program activities. Activities within the intervention program included ropes courses, night trekking, mountain biking, archery, orienteering, and overnight camping. The research protocol takes into account the fact that the specific activities included in the intervention programs were based on age, experience, and other characteristics determined through outdoor education.

Factor analysis included an evaluation of both factor loading and uniqueness. Factor loading is a measure of sample variation and is determined as the square of the outer loading of a given item. Positive factor loading suggests that high scores for a given factor will result in high scores for a given variable. This finding indicated a positive correlation between the factors and the variable for both individual and group actions. The uniqueness of a variable was also evaluated; this calculation denotes interrelatedness of a given factor to others used to create the model. Evaluation of these factors clarified the behaviours of some of the variables associated with the outdoor activities intervention in relation to measures of environmental responsibility evaluated amongst the adolescent participants.

Beginning with perceived information as a factor that included several different variables, evaluation of this parameter resulted in a mean score of 3.75 for the experimental group and 3.47 for the control group. This result implies that the 15 -day intervention program had only a slight impact on this factor. However, if one assigns a factor loading of .7, the results suggest that most of the adolescents who participated in this study ultimately acquired an improved understanding of coastlines and sea pollution, as well as of plants, forests, destruction of indigenous animal populations, and local environmental problems. However, when the factor loading is set at .4 or .5 , which are the standards adopted by most researchers, our results imply that outdoor activities have a positive influence on environmentally conscious perceptions toward genetically modified foods, climate change, global warming, the importance of saving energy, the negative impact of pollution of drinking water, appreciation of positive ways of handling environmental problems, and the use of biologically and ecologically sound products. While tapping into psychosocial mechanisms, outdoor activities are perceived as informative tools that encompass the social environment and that can bring an improved understanding of environmental awareness to adolescents (Cheng and $\mathrm{Wu}, 2015$ ). Such information is 
in itself educational in the sense that it may promote a positive effect on both environmental behaviours and on responses to environmental problems.

Uniqueness scores greater than .6 suggest that each variable may function nearly independently of one another. As such, the results obtained might depend on the precise nature of the outdoor activity included in the intervention program. For instance, trekking would be clearly associated with attributes related to plant, forest, and animal destruction. However, the same trekking activity would not be associated with an appreciation of coastlines and sea pollution, because these variables are not correlated with one another. Based on these observations, it remains significant that perceived information leads to the modification of the psychosocial behaviours which include feelings of guilt, adherence to social norms, internal attribution, and awareness of environmental problems as indicated in the introduction. Modified psychosocial behaviours would subsequently impact environmental responsibility and may compel adolescents to act within their capacity to remedy some of the aforementioned environmental problems.

The second factor considered in this research was cognitive beliefs; these attributes are linked to a number of variables as indicated in the research process. Evaluation of cognitive attributes, as indicated in the conceptual model linked to environmental responsibility, resulted in a mean score of 4.23 for the experimental group and 4.17 for the control group. This again suggests a slight difference between the two groups, and a somewhat higher score overall for those exposed to the intervention program. If factor loading is set at .7 , our results imply that adolescents are aware of the essentials of recycling. However, if factor loading is set at the standard .4 or .5 , our findings imply that adolescents realize the need to participate in replanting of burned forests, the importance of taking part in environmental clean-up events, the significance of learning how to identify products that do not pollute the environment, and the need to avoid water wastage during hand-washing. At the same time, these results also suggest that adolescents understand the importance of further discussion with parents regarding issues focused on the principle of saving energy by turning off household lights when they are not in use. The cognitive attribute reflects an understanding of environmental problems as well as simple and actionable behaviours that correspond to psychosocial mechanisms noted within the immediate social environment. Implications of such an understanding trigger a sense selfresponsibility and concerns that resonate with environmental responsibility (Agan, Kuzey, Acar, \& Acikgoz, 2016). Furthermore, as noted in introduction, the concept of environmental behaviours and the scope of the social environment can engage the cognitive attributes that bridge awareness of educational principles that are not addressed by classroom work. It is conceivable that outdoor activities bring in aspects of the practical world in a manner that permits adolescents to understand and to interpret what they have experienced. Uniqueness amongst the variables is also quite high, with most scores exceeding.6. This finding indicates that the variables associated with cognitive factors and beliefs are not related to one another. However, our findings indicated that scores associated with these variables all rely on the adolescents' participation in specific outdoor activities. For example, an activity such as canoeing on a lake would be more closely associated with the need to participate in clean-up events and not closely associated with actions such as turning off lights when not in use.

Our model and research findings also provide insight into the affective dimension, which is a third critical factor under observation in this study. Affective behaviours reflect brain functions that are associated with emotions, including gratification, jealousy, sexuality, fear, and pleasure, amongst others, and have been attributed to primitive aspects of brain function. This study focused on the affective attributes that were likely to be observed amongst adolescents in relation to environmental responsibility and while experiencing outdoor activities. The mean scores for the affective dimension were 4.17 for the experimental group and 3.99 for the control group. These results imply an affirmative impact of outdoor activities intervention across the two groups. Factor loading set at .7 would imply that most of the adolescents are concerned that people no longer care about the environment, are troubled by unnecessary energy waste, and may become distressed when they see people wasting water in a careless manner. Reducing the factor loading to .4 or .5, which are values preferred by many researchers, implies that adolescents would be irritated by environmental pollution as well as by the 
use of animals in scientific experiments. In addition, most of the participants in this study indicated concern regarding human involvement in the destruction of forests. The affective dimension taps into feelings identified amongst adolescents that are most likely to prompt action-associated behaviours.

The literature review focused on environmental attitudes; these earlier findings resonate well with the affective attributes evaluated in this research. The nature of these attitudes across a given segment of the population would largely determine the nature and scope of the environmental responsibility that is adopted (Abdelkafi \& Tauscher, 2016). As an example, if a population has a negative attitude towards environmental destruction, then it is perceived that individuals within this population would act using all means possible to stop the damage. The variables associated with affective beliefs achieve high scores with respect to uniqueness; this finding implies that a single variable is unlikely to be associated with any of the others due to differences in the outdoor activities interventions. This may relate directly to the specific correlations between the nature of the outdoor activity and the affective dimension adopted by the respondents after participation in the intervention program.

This research also presented significant observations with respect to predisposition for individual action. Predisposition largely describes the tendency to perform or to participate in a specific activity or action. In this context, predisposition for individual action was considered as a factor that was associated with a number of variables. First, mean scores for this factor include 4.18 for the experimental group and 3.82 for the control group. A comparison of the two groups might lead one to conclude that the intervention program had a significant impact with respect to influencing the participants to take action on environmental issues. With factor loading set at .7, our results imply that more adolescents would consider travel by bicycle or by bus with the intent to reduce air pollution associated with the use of personal automobiles. In addition, more or the participants in the experimental group expressed their willingness to take part in home-based recycling. With factor loading set at .4 or .5 , our results suggest that adolescents who participated in this study would recognize the importance of reducing water use while bathing and hand-washing. The factor loading for the predisposition for individual action clearly differentiates amongst action behaviours that result from post-intervention interactions with the social environment. This finding suggests that, upon recognizing a problem, participants assigned to the experimental group were more willing to take steps to remedy the environmental situation at hand than were those who were in the control group. Our research also indicates high scores of uniqueness for this attribute; this finding that implies that the variables associated with this attribute were not closely related to one another due to differences in the outdoor activities.

Lastly, the findings presented in this study provided significant observations with respect to the predisposition for encouraging others. This attribute denotes the likelihood that individuals will advise and/or support others in activities focused on solving environmental problems. The mean scores for the predisposition for encouraging others were 3.12 for the experimental group and 2.92 for the control group. Although these mean scores are lower than those associated with other factors evaluated in this research, they are significantly different from one another. This difference implies that the intervention program had an impact on the participants' predisposition to take action and specifically the predisposition to encourage others to support environmental responsibility. With factor loading set at .7, our results imply that most of the adolescents who participated in the intervention program were willing to go door-to-door to provide others with environmental information. The results of our study also suggest that these participants are also willing to send letters that request help towards solving environmental problems, and to go door-to-door in efforts to encourage others to recycle waste. The predisposition to encourage others promotes group action, which is common activity for the participating adolescents and their peers. Inasmuch as they might want to take action to address environmental problems, adolescents and others typically feel more effective and more confident while working together. This contention focuses on psychosocial behaviours where group action is largely encouraged. Individuals would feel appreciated if others accompany them in taking action towards increased environmental responsibility, given their experiences with outdoor activities. In the course 
of group action, more adolescents might appreciate events connected to the environment and actions related to environmental cleanliness. Interestingly, the scores for uniqueness are relatively low for this attribute compared to the same scores linked to other factors. This result indicates the likelihood of significant relatedness amongst the individual variables. However, the scores still show only limited relatedness with respect to specific outdoor activities, similar to what was observed with other factors.

In summary, the main aim of this research was to evaluate the impact of outdoor activities on attributes associated with environmental responsibility in a cohort of adolescents. The background study and the literature review focused specifically on environmental behaviours, environmental responsibility, psychosocial behaviours, and behavioural mechanisms associated with environmental awareness. The research findings indicated a positive response to all the parameters evaluated. As such, these findings demonstrate the positive impact of outdoor activities on promoting environmental responsibility amongst adolescents who participated in the intervention program.

The observation that participation in outdoor recreational activities has an impact on environmental responsibility can be extended to important applications in other areas. Educational environmental organizations, such as environmental education centers (i.e., the Canadian Conservation Institute) and organized environmental organizations (i.e., the World Wildlife Fund) can benefit from the results of this study and may include similar programs involving outdoor recreation activities in natural environments for their members. Environmental organizations could develop their own bases, centers, and institutes at outdoor facilities to promote participation in activities such as skiing and sailing. These actions might serve to promote their existence, their goals, and the means to achieve these goals, as well as the potential to improve outcomes and results. Programs focused on activities in nature with an overall goal of protecting the environment might be developed by municipalities, prefectures, and educational ministries. Finally, enrichment of environmental education through recreation activities at primary, secondary, high, and environmental, green, forest schools will contribute to the success of these goals and will serve to increase empathy with nature and the environment amongst our future citizens.

\section{Conclusions}

The findings presented in this study support the notion that environmental behaviour is closely related to environmental beliefs, values and attitudes; these factors shape intentions associated with environmental action and ultimately have a profound impact on environmental behaviour. This study contributes to the recognition of individual responsibility and the need to take specific measures to address critical environmental issues. This sense of environmental responsibility should be turned into human action. Encouraging rational environmental action will help to maintain the balance between man and nature and will provide our future citizens with a vision of well-being, an improved quality of life, and a sustainable future.

\section{References}

Abdelkafi, N., \& Tauscher, K. (2016). Business models for sustainability from a system dynamics perspective. Organization E Environment, 29(1), 74-96.

Agan, Y., Kuzey, C., Acar, M.F., \& Acikgoz, A. (2016). The relationships between corporate social responsibility, environmental supplier development, and firm performance. Journal of Cleaner Production, 112(3), $1872-1881$.

Blythe, C., \& Harre, N. (2020). Encouraging transformation and action competence: A Theory of Change evaluation of a sustainability leadership program for high school students. The Journal of Environmental Education, 51(1), 83-96.

Cheng, J.C., \& Monroe, M.C. (2012). Connection to nature: children's affective attitudes toward nature. Environment and Behaviour, 44(1), 31-49.

Cheng, T. M., \& Wu, H. C. (2015). How do environmental knowledge, environmental sensitivity, and place attachment affect environmentally responsible behaviour? An integrated approach for sustainable island tourism. Journal of Sustainable Tourism, 23(4), 557-576. 
Collado, S., Corraliza, J., Staats, H., \& Ruiz, M. (2015). Effect of frequency and mode of contact with nature on children's self-reported ecological behaviors. Journal of Environmental Psychology, 41, 65-73.

Erdogan, M. (2015). The Effect of Summer Environmental Education Program (SEEP) on Elementary School Students' Environmental Literacy. International Journal of Environmental and Science Education, 10(2), 165181.

Harbrow, M. (2019). Visitors as advocates. A review of the relationship between participation in outdoor recreation and support for conservation and the environment. [Online] Available: https://www.doc.govt.nz/globalassets/documents/science-and-technical/sfc333entire.pdf__February 24, 2020)

Heers, M., Van Klaveren, C., Groot, W., \& Maassen van den Brink, H. (2016). Community schools: What we know and what we need to know. Review of Educational Research, 86(4), 1016-1051.

Kukkonen, J., Karkkaine, S., \& Keinonen, T. (2018). Examining the Relationship between Factors Influencing Environmental Behaviours among University Students. Sustainability, 10(11), 4294.

Larson, L.R., Whiting, J.W., \& Green, G.T. (2011). Exploring the influence of outdoor recreation participation on proenvironmental behaviour in a demographically diverse population. Local Environment, 16(1), 67-86.

Lubans, D., Richards, J., Hillman, C., Faulkner, G., Beauchamp, M., Nilsson, M., \& Biddle, S. (2016). Physical activity for cognitive and mental health in youth: a systematic review of mechanisms. Pediatrics, 138(3), e20161642.

Mackenzie, S.H., Son, J.S., \& Eitel, K. (2018). Using outdoor adventure to enhance intrinsic motivation and engagement in science and physical activity: an exploratory study. Journal of Outdoor Recreation and Tourism, 21, $76-86$.

Palmberg, I.E. \& Kuru, J. (2000). Outdoor activities as a basis for environmental responsibility. Journal of Environmental Education, 31(4), 32-36.

Rantala, Q., \& Puhakka, R. (2020). Engaging with nature: nature affords well-being for families and young people in Finland. Children's Geographies, 18(4), 490-503.

Reis, R.S., Salvo, D., Ogilvie, D., Lambert, E.V., Goenka, S., Brownson, R.C., \& Lancet Physical Activity Series 2 Executive Committee (2016). Scaling up physical activity interventions worldwide: stepping up to larger and smarter approaches to get people moving. The Lancet, 388(10051), 1337-1348.

Sathe, N. (2018). Factors Affecting Adolescent Adjustment and Scholastic Performance. International Journal of Innovative Knowledge Concepts, 6(5), 85-93.

Singh, S., Goyal, R., \& Jain, A. (2017). The Urban Environmental Crisis in India. New Initiatives in Safe Water and Waste Management. Cambridge Scholars Publishing.

Sivek, D.K. (2002), Environmental sensitivity among Wisconsin high school students. Environmental Education Research, 8(2), 155-170.

Stevenson, K.T., Peterson, M.N., Carrier, S.J., Strnad, R.L., Bondell, H.D., Kirby-Hathaway, T., \& Moore, S.E. (2014). Role of significant life experiences in building environmental knowledge among middle school students. Journal of Environmental Education, 45(3), 163-177.

Taylor, L.G., Clark, A.F., \& Gilliland, J.A. (2018). Context Matters: Examining children's perceived barriers to physical activity across varying Canadian environments. Health and Place, 54, 221-228.

Thatcher, A., Waterson, P., Todd, A., \& Yeow, P.H. (2018). The Way Forward for Human Factors/Ergonomics and Sustainability. In Congress of the International Ergonomics Association (pp. 616-625). Springer, Cham.

Zafeiroudi, A., \& Hatzigeorgiadis, A. (2013). Environmental Responsibility in Secondary School Students: Development, Validation \& Relationships between Environmental Scales. Inquiries in Sport E Physical Education, 12(3), 253-264.

Zafeiroudi, A., \& Hatzigeorgiadis, A. (2014a). The effects of an outdoor pursuit's intervention program on adolescents' environmental beliefs. International Journal on Advances in Education Research, 1(3), 106-118.

Zafeiroudi, A., \& Hatzigeorgiadis, A. (2014b). Validation of the Responsible Environmental Behaviour Scale and relationships with participation in outdoor activities. International Journal of Sport Management, Recreation and Tourism, 13, 20-37. 